\title{
Comprehensive evaluation of a somatostatin-based radiolabelled antagonist for diagnostic imaging and radionuclide therapy
}

\author{
Xuejuan Wang • Melpomeni Fani • Stefan Schulz • \\ Jean Rivier • Jean Claude Reubi • Helmut R. Maecke
}

Received: 1 March 2012 / Accepted: 9 August 2012 /Published online: 29 August 2012

(C) Springer-Verlag 2012

\begin{abstract}
Purpose Targeting of tumours positive for somatostatin receptors (sst) with radiolabelled peptides is of interest for tumour localization, staging, therapy follow-up and targeted radionuclide therapy. The peptides used clinically are exclusively agonists, but recently we have shown that the radiolabelled somatostatin-based antagonist ${ }^{111}$ In-DOTA-sst2ANT may be preferable to agonists. However, a comprehensive study of this radiolabelled antagonist to determine its significance was lacking. The present report describes the evaluation of this novel antagonist labelled with ${ }^{111}$ In and ${ }^{177} \mathrm{Lu}$ in three different tumour models.

Methods Radiopeptide binding, internalization and dissociation studies were performed using cells expressing HEK293rsst $_{2}$. Biodistribution studies were performed in HEK293rsst $_{2}$, HEK293-hsst 2 and HEK293-rsst ${ }_{3}$ xenografted mice.
\end{abstract}

X. Wang $\cdot$ M. Fani $\cdot$ H. R. Maecke

Division of Radiological Chemistry, University Hospital Basel, Basel, Switzerland

\section{S. Schulz}

Department of Pharmacology and Toxicology, Jena University Hospital - Friedrich Schiller University Jena,

Jena, Germany

J. Rivier

The Clayton Foundation Laboratories for Peptide Biology,

The Salk Institute for Biological Studies,

La Jolla, CA, USA

J. C. Reubi

Division of Cell Biology and Experimental Cancer Research, Institute of Pathology, University of Bern,

Bern, Switzerland

Present Address:

H. R. Maecke ( $\square)$

Department of Nuclear Medicine, University Hospital Freiburg,

Hugstetterstrasse 55,

79106 Freiburg, Germany

e-mail: helmut.maecke@uniklinik-freiburg.de
Results Saturation binding analysis confirmed earlier $\mathrm{IC}_{50}$ data for ${ }^{111 / n a t}$ In-DOTA-sst2-ANT and showed similar affinity of ${ }^{177 / n a t}$ Lu-DOTA-sst2-ANT for the sst $_{2}$. Only low internalization was found in cell culture $(6.68 \pm 0.06 \%$ at $4 \mathrm{~h})$, which was not unexpected for an antagonist, and this could be further reduced by the addition of sucrose. No internalization was observed in HEK293 cells not expressing sst. Both results indicate that the internalization was specific. ${ }^{111}$ In-DOTAsst2-ANT and ${ }^{177}$ Lu-DOTA-sst2-ANT were shown to target tumour xenografts expressing the rat and the human sst $_{2}$ receptor with no differences in their uptake or pharmacokinetics. The uptake in rsst $_{2}$ and hsst w $_{2}$ as high (about $30 \% \mathrm{IA} / \mathrm{g} 4 \mathrm{~h}$ after injection) and surprisingly long-lasting (about 20-23 $\% \mathrm{IA} / \mathrm{g} 24 \mathrm{~h}$ after injection). Kidney uptake was blocked by approximately $50 \%$ by lysine or Gelofusine.

Conclusion These results indicate that radiolabelled somatostatin-based antagonists may be superior to corresponding agonists. The long tumour retention time of ${ }^{177}$ Lu-DOTA-sst2-ANT indicates that this new class of compounds is of relevance not only in diagnostic imaging but also in targeted radionuclide therapy of sst-positive tumours.

Keywords Somatostatin · Antagonist · Imaging · Radionuclide therapy

\begin{tabular}{|c|c|}
\hline \multicolumn{2}{|c|}{ Abbreviations } \\
\hline HEK & Human embryonic kidney \\
\hline DOTA & $\begin{array}{l}\text { 1,4,7,10-tetraazacyclododecane-1,4, } \\
\text { 7,10-tetracetic acid }\end{array}$ \\
\hline CB-TE2A & $\begin{array}{l}\text { 4,11-bis(carboxymethyl)-1,4,8, } \\
\text { 11-tetraazabicyclo[6.6.2]hexadecane }\end{array}$ \\
\hline DMEM & Dulbecco's modified Eagle's medium \\
\hline BSA & Bovine serum albumin \\
\hline FBS & Fetal bovine serum \\
\hline PBS & Phosphate-buffered saline \\
\hline HEPES & $\begin{array}{l}\text { 2-[4-(2-Hydroxyethyl)piperazin-1-yl] } \\
\text { ethanesulphonic acid }\end{array}$ \\
\hline
\end{tabular}




\section{Introduction}

Receptors for regulatory peptides have been found to be overexpressed in a variety of human tumours. This led to the idea to develop radiolabelled peptides and use them in sensitive imaging modalities such as single photon emission computed tomography (SPECT) and positron emission tomography (PET) for tumour imaging, and to design cytotoxic agents that could be used in targeted cancer treatment. The prototypes of these peptides are based on somatostatin $[1,2]$, targeting somatostatin receptors (sst), most importantly the subtype $2\left(\mathrm{sst}_{2}\right)$. The consensus was that successful in vivo tumour targeting requires the use of receptor agonists as they are internalized and therefore have a long retention time in the tumour, an important prerequisite for targeted radionuclide therapy if longer-lived $\beta^{-}$emitters such as ${ }^{90} \mathrm{Y}$ or ${ }^{177} \mathrm{Lu}$ are being used.

This hypothesis appeared to be supported by recent data reported by Storch et al. [3]. They have shown that there is a good correlation between tumour uptake as well as uptake in sst-positive organs (e.g. pancreas) and the rate of internalization into $\mathrm{sst}_{2}$-expressing cells in culture. Fani et al. [4] have shown that high affinity but slowly internalizing bicyclic somatostatin agonists have relatively low uptake and fast washout from sst-positive tumours in vivo. In addition, Ginj et al. [5] studied a series of radiolabelled DOTAconjugated pansomatostatin analogues (carbocyclic octapeptides) which showed high affinity for $\mathrm{sst}_{1}-\mathrm{sst}_{5}$ and agonistic potency if studied using an adenylate cyclase activity assay. The agonistic properties of the pansomatostatin peptides were tested in terms of their effect on forskolin-stimulated cAMP accumulation. $\mathrm{EC}_{50}$ values obtained from this assay were shown to be comparable with those of the natural somatostatin-28 (SS-28). Surprisingly, these radioligands showed efficient internalization only in cell lines expressing sst $_{3}$; inefficient or no internalization was found in cell lines expressing sst $_{2}$ and sst $_{5}$. As a consequence in a dual tumour model only sst ${ }_{3}$-expressing tumours showed high and persistent tumour uptake, whereas $\mathrm{sst}_{2}$-expressing tumours showed low uptake and fast washout [5]. Contrary to these results, we have recently found that potent radiolabelled $\mathrm{sst}_{2}$ and $\mathrm{sst}_{3}$ antagonists show higher in vivo tumour uptake as well as tumour-to-normal tissue ratios than radiolabelled agonists of similar or even higher receptor affinity [6]. The ${ }^{111} \mathrm{In}$-labelled antagonist DOTA-sst2-ANT (where sst2-ANT is 4- $\mathrm{NO}_{2}$ Phe-c(D-Cys-Tyr-D-Trp-Lys-Thr-Cys)-D-Tyr- $\mathrm{NH}_{2}$ ) showed high tumour uptake in HEK-sst ${ }_{2}$ xenografts and surprisingly long retention, despite the fact that very little internalization was detected [6].

Somewhat contrary to these data, Wadas et al. concluded from their studies with two ${ }^{64} \mathrm{Cu}$-labelled somatostatin-based peptides that the agonist ${ }^{64} \mathrm{Cu}-\mathrm{CB}$ TE2A-TATE shows somewhat higher tumour uptake than ${ }^{64} \mathrm{Cu}$-CB-TE2A-sst2-ANT despite the fact that there was about a 14-fold difference in receptor binding sites in their rat pancreatic AR42J cell line in favour of the antagonist [7], similar to our findings in a transfected cell line HEK293rsst $_{2}[6]$. The much lower affinity of the antagonist compared to the agonist may be the reason for these findings.

We therefore decided to study this radiopeptide more thoroughly, and extended the pharmacokinetics to later time-points in order to investigate the potency of these new radiolabelled antagonists as therapeutic radiopharmaceuticals. Therefore we also extended the study using ${ }^{177} \mathrm{Lu}$ and studied the biodistribution of ${ }^{177} \mathrm{Lu}$-DOTA-sst2-ANT to determine if the radiometal has an influence on the pharmacokinetics of this new class of radiopeptides, which is possible due to the potential differences in the geometry of the radiometal complexes. We also looked into the possibility of blocking the tubular cell-mediated kidney uptake of antagonistic radiopeptides with agents that are successfully used clinically with the corresponding radiolabelled agonists. Finally, we studied ${ }^{111}$ In-DOTA-sst2-ANT in a different tumour model, namely a subcutaneous mouse xenograft model with HEK293-cell lines expressing the human sst $_{2}$ receptor rather than the rat receptor exclusively used in the earlier study to exclude species differences as found with gastrin-releasing peptide receptor targeting bombesin derivatives [8].

\section{Materials and methods}

Preparation of radiotracers

DOTA-sst2-ANT was synthesised as described previously $[9,10]$. The ${ }^{111}$ In- and ${ }^{177} \mathrm{Lu}-\mathrm{DOTA}-$ sst2-ANT were prepared after incubation of $5 \mu \mathrm{g}$ DOTA-sst2-ANT in $300 \mu \mathrm{L}$ sodium acetate buffer $(0.4 \mathrm{M}, \mathrm{pH} 5)$ with ${ }^{111} \mathrm{InCl}_{3}$ or ${ }^{177} \mathrm{LuCl}_{3}(37-74 \mathrm{MBq})$, respectively, at $95^{\circ} \mathrm{C}$ for $30 \mathrm{~min}$. Quality control was performed by RP-HPLC, as previously described [4].

For all in vitro studies excess of ${ }^{\text {nat }} \mathrm{InCl}_{3} \cdot 5 \mathrm{H}_{2} \mathrm{O}$ was added after the formation of ${ }^{111} \mathrm{In}$-DOTA-sst2-ANT and the reaction mixture was incubated at $95^{\circ} \mathrm{C}$ for an additional $30 \mathrm{~min}$ to form homogeneous ${ }^{111 / \text { nat }}$ In-DOTA-sst2-ANT solution. For the in vivo studies the solutions of ${ }^{111}$ In-DOTA-sst2ANT and ${ }^{177} \mathrm{Lu}$-DOTA-sst2-ANT were prepared by dilution with $0.9 \% \mathrm{NaCl} / 0.1 \%$ BSA.

\section{Cell lines}

Cells of the transfected HEK293 cell line stably expressing the rat $\mathrm{sst}_{2}$ and $\mathrm{sst}_{3}$ receptors (HEK293-rsst ${ }_{2}$ and HEK293rsst $_{3}$ ) and the human sst $_{2}$ receptor (HEK293-hsst ${ }_{2}$ ) were used in the in vitro and in vivo studies. HEK293 cells 
without transfection were used as negative control. All transfected cells were cultured at $37{ }^{\circ} \mathrm{C}$ in an atmosphere containing $5 \% \mathrm{CO}_{2}$ in DMEM containing $10 \% \mathrm{FBS}$, $100 \mathrm{U} / \mathrm{mL}$ penicillin, $100 \mu \mathrm{g} / \mathrm{mL}$ streptomycin and $500 \mu \mathrm{g} / \mathrm{mLG}$ 418. The nontransfected HEK293 cells were cultured in the same medium without G418. Culture reagents were supplied by BioConcept (Allschwil, Switzerland).

\section{Determination of the sst affinity}

Affinity was determined on HEK293-rsst ${ }_{2}$ cell membranes. Briefly, the cells were rinsed with cold PBS (pH7.4), resuspended in homogenization buffer $(20 \mathrm{mM}$ Tris- $\mathrm{HCl}, 1 \mathrm{mM}$ ethylenediaminetetraacetic acid, $0.25 \mathrm{M}$ sucrose, $\mathrm{pH} 7.6$ ) with protease and phosphatase inhibitors $(1 \mathrm{mg} / \mathrm{mL}$ bacitracin, $0.1 \mathrm{mg} / \mathrm{mL}$ soybean trypsin inhibitor, $0.125 \mathrm{mg} / \mathrm{mL}$ phenylmethylsulphonyl fluoride) and lysed using a homogenizer on ice. After low-speed centrifugation $(500 \mathrm{~g}, 5 \mathrm{~min})$, the supernatants were collected and centrifuged at 20,000 $\mathrm{g}$ for $50 \mathrm{~min}$ to separate the cell membranes. Membranes were suspended in HEPES buffer using a homogenizer. Protein content was determined using the Bradford reagent (BioRad Laboratories, Marnes La Coquette, France) using BSA as a standard.

Saturation binding studies were performed in quadruplicate in 96-well plates (10 $\mu \mathrm{g}$ cell membrane per well) using a MultiScreen ${ }_{\text {HTS }}^{\circledR} 96$ assay system (Millipore, Billerica, MA). Increasing concentrations of ${ }^{111 / n a t}$ In-DOTA-sst2ANT and ${ }^{177 / n a t}$ Lu-DOTA-sst2-ANT, ranging from 0.05 $\mathrm{nM}$ to $100 \mathrm{nM}$, were prepared with the binding buffer (150 mM HEPES, $10 \mathrm{mM} \mathrm{MgCl} 2,14 \mathrm{mg} / \mathrm{L}$ bacitracin, $0.3 \% \mathrm{w} / \mathrm{v}$ BSA, $\mathrm{pH} 7.6)$. After the addition of the radiopeptide to the membranes with or without a 1,000 -fold excess of DOTA-sst2-ANT to determine nonspecific binding, the plates were shaken for $2 \mathrm{~h}$ at room temperature. The membranes were then washed three times with binding buffer and measured in a $\gamma$-counter (Packard, Cobra II). Specific binding was calculated by subtracting nonspecific from total binding at each radiopeptide concentration. Dissociation constants $\left(K_{\mathrm{d}}\right)$ and maximum numbers of binding sites $\left(B_{\max }\right)$ values were calculated from Scatchard plots using Origin 7.5 software (Microcal Software, Northampton, MA).

Internalization studies

Internalization of ${ }^{111 / n a t}$ In-DOTA-sst2-ANT was investigated under four different conditions. Initially, the specific internalization of ${ }^{111 / \text { nat }}$ In-DOTA-sst2-ANT was determined in HEK293-rsst ${ }_{2}$ cells at $37{ }^{\circ} \mathrm{C}$. The same experiment was repeated with HEK293-rsst2 cells pretreated with $0.5 \mathrm{M}$ sucrose for $30 \mathrm{~min}$ before the addition of the radiopeptide to determine whether the internalization was via receptormediated endocytosis. The experiment was also carried out in HEK293 cells without receptors and finally the experiment was performed at $4{ }^{\circ} \mathrm{C}$ to prevent internalization.

All experiments were performed in six-well plates. Briefly, approximately $2.5 \mathrm{pmol}$ of ${ }^{111 / \mathrm{nat}} \mathrm{In}$-DOTA-sst2-ANT was added to the medium and the cells $\left(10^{6}\right.$ cells per well $)$ and incubated (in triplicate) for $0.5,1,2$ and $4 \mathrm{~h}$ at $37^{\circ} \mathrm{C}$ in an atmosphere containing $5 \% \mathrm{CO}_{2}$ (or $4{ }^{\circ} \mathrm{C}$ in the last experimental condition). Nonspecific surface-bound and internalized radiopeptide were determined in the presence of a 1,000-fold excess of DOTA-sst2-ANT. The final volume was $1.5 \mathrm{~mL}$ per well. At the indicated time-points the cellular uptake was stopped by removal of the medium followed by washing the cells with ice-cold PBS (pH7.4). Cells were then treated for $5 \mathrm{~min}$ (three times) with glycine buffer $(0.05 \mathrm{~mol} / \mathrm{L}$ glycine solution, $\mathrm{pH} 2.8)$ to distinguish between cell surface-bound radiopeptide (acid-releasable) and internalized radiopeptide (acid-resistant). Finally, the cells were detached from the plates by incubation with $1 \mathrm{M} \mathrm{NaOH}$ for $10 \mathrm{~min}$ at $37^{\circ} \mathrm{C}$. The radioactivity of all fractions was measured in the $\gamma$-counter.

\section{Dissociation studies}

The dissociation of ${ }^{111 / n a t}$ In-DOTA-sst2-ANT was studied in HEK293-rsst ${ }_{2}$ cells under two different design settings. In the first study, approximately $2.5 \mathrm{pmol}$ of $111 /$ nat In-DOTA-sst2-ANT was added to the cells followed by incubation for $3 \mathrm{~h}$ on ice. The low temperature prevents internalization while the long incubation time ensures equilibrium. After $3 \mathrm{~h}$ the unbound radiopeptide was rinsed off with cold medium and the cells were treated with $0.9 \mathrm{~mL}$ of prewarmed medium (DMEM containing $1 \%$ FBS) along with $0.1 \mathrm{~mL}$ of DOTA-sst2-ANT (50 or $250 \mathrm{pmol}$ ) or $0.1 \mathrm{~mL}$ PBS. The six-well plates were then immediately transferred to $37{ }^{\circ} \mathrm{C}$. After $5 \mathrm{~min}$ the whole medium, which contained the dissociated radiopeptide, was quickly removed for quantification. The same amount of medium with or without DOTA-sst2-ANT, as described above, was added to the cells and incubated until the next timepoint. This procedure was repeated for all investigated time-points $(5,10,15,30$ and $60 \mathrm{~min})$. The surfacebound fractions were obtained as described above and quantified in the $\gamma$-counter.

In the second study, HEK293-rsst ${ }_{2}$ cells were treated with different amounts of ${ }^{111 / n a t}$ In-DOTA-sst2-ANT $(0.2$, $0.5,1.0$ and $2.5 \mathrm{pmol}$ ) for $3 \mathrm{~h}$ on ice and the unbound radioligand was then rinsed off. Fresh pre-warmed medium $(1 \mathrm{~mL})$ was added to each well followed by incubation for $5,10,15,30,40$ and $60 \mathrm{~min}\left(37^{\circ} \mathrm{C}\right.$ in an atmosphere containing $5 \% \mathrm{CO}_{2}$ ). At each time-point 
the medium, which contained the dissociated radioligand, was quickly removed for quantification and fresh medium was added.

Animal models

All animal studies were performed in accordance with the guidelines of Swiss regulations (approval 789). Athymic female nude mice at 4-5 weeks of age were implanted subcutaneously (s.c.) in the right shoulder with $10^{7}$ HEK293-rsst $_{2}$ or HEK293-hsst 2 cells freshly suspended in sterile PBS. A dual tumour model was also used in which mice were implanted with $10^{7}$ HEK293-rsst $2_{2}$ in the right shoulder and $10^{7}$ HEK293rsst $_{3}$ in the left shoulder. As HEK293-rsst 3 cells grow somewhat faster, they were implanted 3 days later than the HEK293-rsst 2 cells. Tumours were allowed to grow for approximately 2 weeks to reach a diameter of $8-10 \mathrm{~mm}$.

Biodistribution of ${ }^{111}$ In-DOTA-sst2-ANT and ${ }^{177}$ Lu-DOTA-sst2-ANT

Nude mice were injected into the tail vein with $0.1 \mathrm{~mL}$ of a 10-pmol solution of ${ }^{111}$ In- or ${ }^{177}$ Lu-DOTA-sst2-ANT (approximately $0.2 \mathrm{MBq}$ ). For the determination of nonspecific uptake in the tumour or $\mathrm{sst}_{2}$ receptor-positive organs, a group of mice were injected with a mixture of $10 \mathrm{pmol}$ radiopeptide with $20 \mathrm{nmol}$ DOTA-sst2-ANT. At preselected time-points mice were killed and dissected. The biodistribution of ${ }^{111}$ In-DOTA-sst2-ANT was studied in the dual HEK293-rsst 2 /HEK293-rsst 3 xenografted mice at $30 \mathrm{~min}$ and 4, 24, 48 and $72 \mathrm{~h}$ after injection (p.i.) and in HEK293-hsst ${ }_{2}$ xenografted mice at 1, 4, 24, 48 and $72 \mathrm{~h}$ p.i. The biodistribution of ${ }^{177}$ Lu-DOTA-sst2-ANT was studied in HEK293-rsst 2 xenografted mice at 4, 24, 48 and $72 \mathrm{~h}$ p.i. Organs of interest were collected, rinsed of excess blood, weighed and counted in the $\gamma$-counter together with the injection standards. The percentage of injected activity per gram tissue $(\% \mathrm{IA} / \mathrm{g})$ was calculated for each tissue.

\section{Blocking of renal uptake}

The plasma expander Gelofusine, a succinylated gelatin solution $(40 \mathrm{mg} / \mathrm{mL})$ and a lysine solution containing $200 \mathrm{mg} / \mathrm{mL}$ in PBS were used in an attempt to reduce the kidney uptake of ${ }^{111}$ In-DOTA-sst2-ANT. HEK293-rsst ${ }_{2}$ xenografted mice were intravenously injected with Gelofusine $(4 \mathrm{mg})$ or lysine $(20 \mathrm{mg})$ into the tail vein. Two other injected doses were also used $(6 \mathrm{mg}$ of Gelofusine and $30 \mathrm{mg}$ of lysine per animal). ${ }^{111}$ In-DOTA-sst2-ANT $(0.1 \mathrm{~mL}$ of a 10 -pmol solution, approximately $0.2 \mathrm{MBq})$ was injected 5 min later. Animals were killed 24 h p.i. and biodistribution studies were performed as described above.
Statistical analysis

Values are presented as means $\pm \mathrm{SD}$. Student's $t$ test was used to determine statistical significance. The significance level was set at 0.05 .

\section{Results}

Chemistry and radiochemistry

The synthesis and characterization of the DOTA-sst2-ANT was according to published procedures $[4,9,10]$. The purity of the peptide as determined by HPLC was $>97 \%$. ${ }^{111}$ Inand ${ }^{177} \mathrm{Lu}$-DOTA-sst2-ANT were prepared in high labelling yield and radiochemical purity ( $>95 \%)$ at specific activities of $10-20 \mathrm{MBq} / \mathrm{nmol}$.

In vitro binding and internalization studies

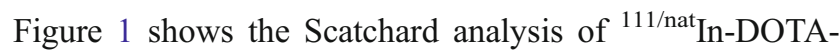
sst2-ANT on HEK-rsst ${ }_{2}$ cell membranes. The calculated dissociation constant $\left(K_{\mathrm{d}}\right)$ was $5.78 \pm 0.64 \mathrm{nM}$ and the calculated maximum number of binding sites $\left(B_{\max }\right)$ was $37.6 \pm 1.2 \mathrm{pmol} / \mathrm{mg}$. Statistical analysis showed a significant correlation (Pearson correlation coefficient $r=-0.9726$ and $\left.R^{2}=0.9460\right)$. The dissociation constant of ${ }^{177 / n a t}$ Lu-DOTAsst2-ANT was similar $\left(K_{\mathrm{d}}=8.16 \pm 0.90 \mathrm{nM}\right)$.

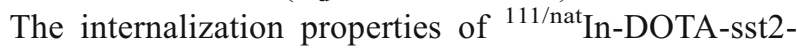
ANT were initially studied after incubation of HEK293rsst $_{2}$ cells with the radiopeptide at $37{ }^{\circ} \mathrm{C}$ in the absence or presence of a 1,000-fold excess of DOTA-sst2-ANT

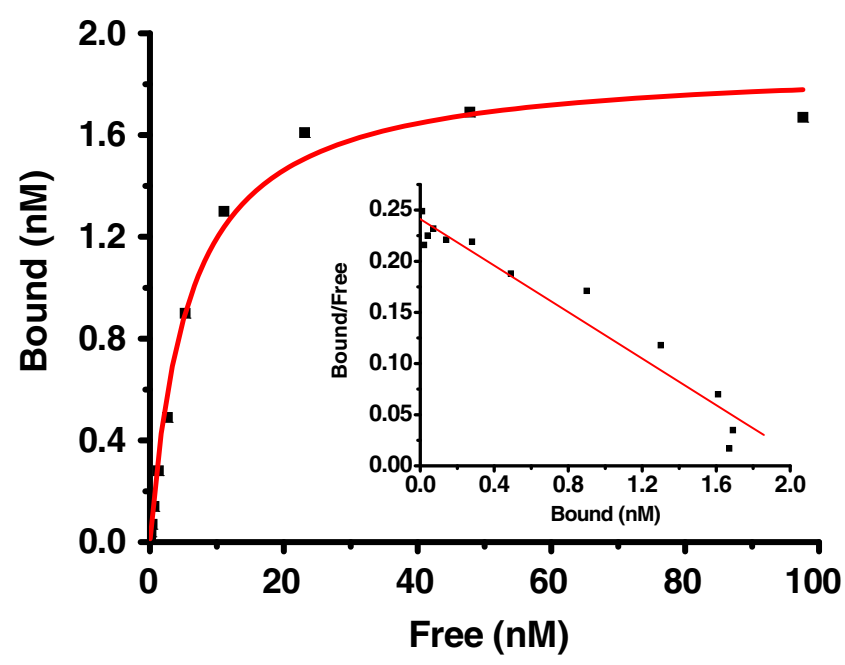

Fig. 1 Saturation binding study on HEK293-rsst ${ }_{2}$ cell membranes, using increased concentrations of ${ }^{111 / n a t}$ In-DOTA-sst2-ANT, ranges from 0.05 to $100 \mathrm{nM}$. The dissociation constants $\left(K_{\mathrm{d}}\right)$ and maximum numbers of binding sites $\left(\mathrm{B}_{\max }\right)$ were calculated using Origin 7.5 software. All data are from quadruplicate experiments 


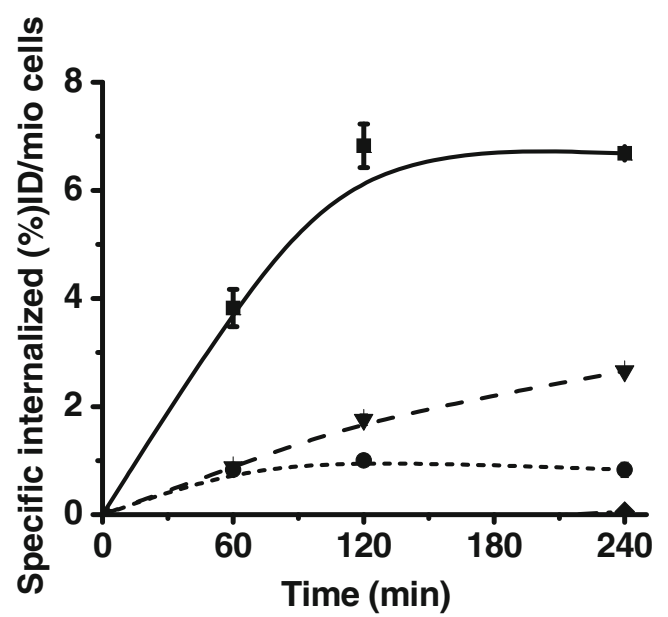

Fig. 2 Specific internalization rates of ${ }^{111 / n a t}$ In-DOTA-sst2-ANT into HEK293-rsst ${ }_{2}$ cells incubated at $37{ }^{\circ} \mathrm{C}$ (squares), into HEK293-rsst cells pretreated with $0.5 \mathrm{M}$ sucrose and incubated at $37^{\circ} \mathrm{C}$ (triangles), into HEK293-rsst ${ }_{2}$ cells incubated at $4{ }^{\circ} \mathrm{C}$ (circles) and into HEK293 cells incubated at $37{ }^{\circ} \mathrm{C}$ (diamond). The results are expressed as specific internalization (percentage of dose added to 1 million cells). All data are from three independent experiments performed in triplicate

(nonspecific uptake). Specific internalization over time is

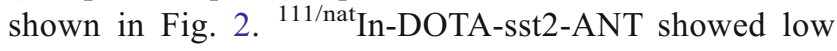
internalization. At $4 \mathrm{~h}$, only $6.68 \pm 0.06 \%$ of the radiopeptide was internalized, which corresponds to the plateau, while $30.63 \pm 0.28 \%$ was bound on the cell surface (data

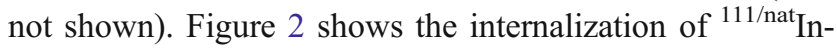
DOTA-sst2-ANT under the various experimental settings. Pretreatment of the cells with hypertonic sucrose $(0.5 \mathrm{M})$ or incubation at $4{ }^{\circ} \mathrm{C}$ significantly reduced or even prevented

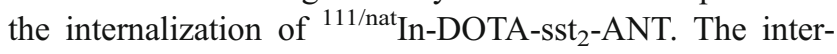
nalized fraction at $4 \mathrm{~h}$ dropped from $6.68 \pm 0.06 \%\left(37^{\circ} \mathrm{C}\right)$ to $2.66 \pm 0.05 \%$ when the cells were pretreated with sucrose

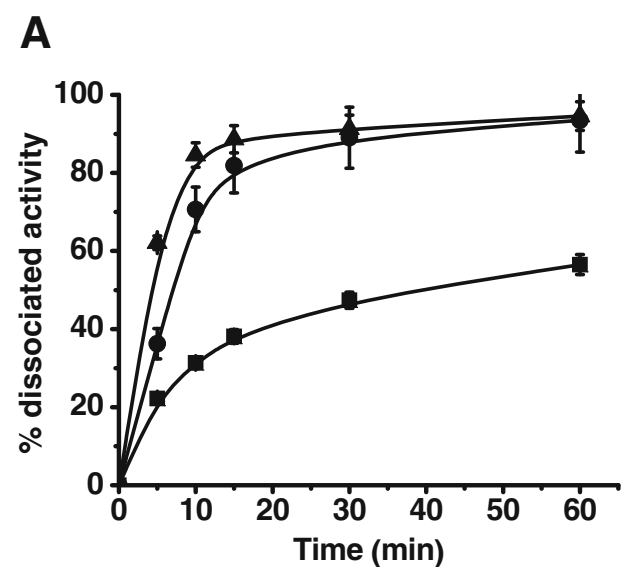

Fig. 3 Dissociation of ${ }^{111 / n a t}$ In-DOTA-sst2-ANT from HEK293-rsst 2 cells at $37^{\circ} \mathrm{C}$. a $2.5 \mathrm{pmol}$ of ${ }^{111 / \text { nat In-DOTA-sst2-ANT was allowed to }}$ bind to the receptors on the cell surface at $4{ }^{\circ} \mathrm{C}$ and after removal of the unbound radiopeptide, the cells were treated with PBS (squares), or with $50 \mathrm{pmol}$ (circles) or $250 \mathrm{pmol}$ (triangles) DOTA-sst2-ANT at $37^{\circ}$ C. b $0.2 \mathrm{pmol}$ (diamonds), $0.5 \mathrm{pmol}$ (triangles), $1.0 \mathrm{pmol}$ (squares) and and to $0.83 \pm 0.14 \%$ when the cells were incubated at $4{ }^{\circ} \mathrm{C}$. No internalization of ${ }^{111 / \text { nat }}$ In-DOTA-sst ${ }_{2}$-ANT was found in the nontransfected HEK293 cells.

Dissociation studies

The disappearance of ${ }^{111 / \mathrm{nat}}$ In-DOTA-sst2-ANT from the receptors was evaluated in HEK293-rsst ${ }_{2}$ cells which were treated with PBS, or with $50 \mathrm{pmol}$ or $250 \mathrm{pmol}$ of DOTA-sst2-ANT, as described in Materials and methods. The dissociation profiles are shown in Fig. 3a. A relatively slow release of the cell-associated radioactivity was found if no DOTA-sst2-ANT was added to the fresh medium (cells treated with PBS); $56.5 \pm 2.6 \%$ of the radiopeptide was detected in the medium after $60 \mathrm{~min}$. The addition of $50 \mathrm{pmol}$ or $250 \mathrm{pmol}$ of DOTA-sst2-ANT to the medium (to inhibit rebinding of the radiopeptide) led to an irreversible dissociation with a $t_{1 / 2}$ of about $10 \mathrm{~min}$. Within the first $15 \mathrm{~min}$, $81.9 \pm 7.0 \%$ and $88.6 \pm 3.5 \%$ of the radiopeptide had dissociated from the $\mathrm{sst}_{2}$ receptors when $50 \mathrm{pmol}$ and 250 pmol DOTA-sst2-ANT was added, respectively, and at $60 \mathrm{~min}$ almost all the radiopeptide has dissociated $(93.5 \pm 4.1 \%$ and $94.6 \pm 3.7 \%$, respectively; Fig. 3a).

We also determined whether the different concentrations of ${ }^{111 / n a t}$ In-DOTA-sst2-ANT had any influence on the dissociation rate of the radiopeptide from the receptors. The results are summarized in Fig. 3b. No significant differences were observed in the dissociation rates of ${ }^{111 / \mathrm{nat} I n-D O T A-}$ sst2-ANT in the range of 0.2 to $1.0 \mathrm{pmol} / \mathrm{mL}$. However, significantly higher values were found over time when the cells were treated with the highest concentration $(2.5 \mathrm{pmol} /$ $\mathrm{mL}$ ) of ${ }^{111 /{ }^{n a t}}$ In-DOTA-sst 2 -ANT.

\section{B}

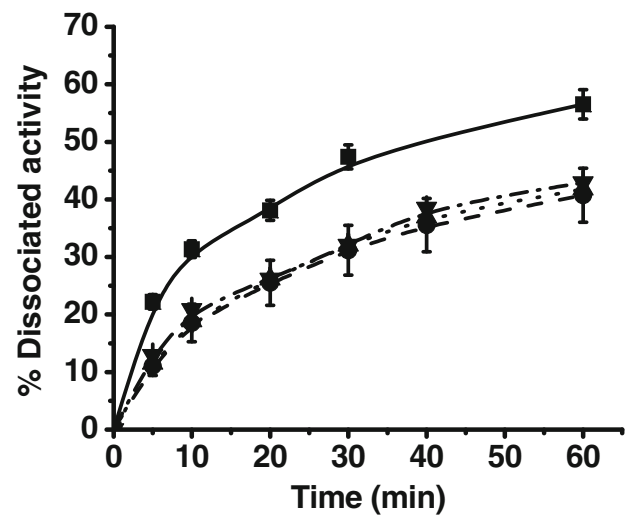

$2.5 \mathrm{pmol}$ (circles) ${ }^{111 / \mathrm{nat}}$ In-DOTA-sst2-ANT was allowed to bind to the receptors on the cell surface at $4{ }^{\circ} \mathrm{C}$ and after removal of the unbound radiopeptide, the cells were incubated with fresh medium at $37^{\circ} \mathrm{C}$. At each time-point the percentage of radiopeptide which had dissociated from the receptors was calculated and plotted 
Table 1 Biodistribution (mean \pm SD $\%$ IA $/ \mathrm{g}, n=4)$ of ${ }^{111}$ In-DOTAsst2-ANT in nude mice simultaneously bearing HEK-rsst ${ }_{2}$ and $\mathrm{HEK}_{\text {-rsst }}$ tumours
${ }^{\mathrm{a} C o i n j e c t i o n ~ o f ~} 20 \mathrm{nmol}$ DOTAsst2-ANT.

\begin{tabular}{|c|c|c|c|c|c|c|}
\hline Organ & $30 \mathrm{~min}$ & $4 \mathrm{~h}$ & 4 h blocking ${ }^{a}$ & $24 \mathrm{~h}$ & $48 \mathrm{~h}$ & $72 \mathrm{~h}$ \\
\hline Blood & $2.76 \pm 0.19$ & $0.14 \pm 0.03$ & $0.13 \pm 0.01$ & $0.05 \pm 0.01$ & $0.01 \pm 0.00$ & $0.01 \pm 0.00$ \\
\hline Heart & $1.23 \pm 0.05$ & $0.11 \pm 0.03$ & $0.08 \pm 0.01$ & $0.04 \pm 0.00$ & $0.03 \pm 0.01$ & $0.02 \pm 0.01$ \\
\hline Liver & $1.74 \pm 0.18$ & $0.43 \pm 0.07$ & $0.49 \pm 0.03$ & $0.32 \pm 0.02$ & $0.26 \pm 0.02$ & $0.24 \pm 0.05$ \\
\hline Spleen & $1.67 \pm 0.23$ & $0.23 \pm 0.04$ & $0.21 \pm 0.02$ & $0.15 \pm 0.02$ & $0.11 \pm 0.01$ & $0.12 \pm 0.03$ \\
\hline Lung & $6.79 \pm 2.01$ & $0.46 \pm 0.22$ & $0.32 \pm 0.04$ & $0.17 \pm 0.01$ & $0.10 \pm 0.03$ & $0.04 \pm 0.03$ \\
\hline Kidney & $22.92 \pm 2.62$ & $10.50 \pm 1.00$ & $9.67 \pm 1.38$ & $7.38 \pm 0.09$ & $4.33 \pm 0.56$ & $2.96 \pm 0.35$ \\
\hline Stomach & $7.82 \pm 2.03$ & $0.61 \pm 0.18$ & $0.19 \pm 0.07$ & $0.25 \pm 0.06$ & $0.16 \pm 0.02$ & $0.14 \pm 0.02$ \\
\hline Intestine & $1.72 \pm 0.25$ & $0.16 \pm 0.03$ & $0.15 \pm 0.03$ & $0.08 \pm 0.03$ & $0.05 \pm 0.02$ & $0.03 \pm 0.01$ \\
\hline Adrenals & $4.74 \pm 3.00$ & $0.49 \pm 0.12$ & $0.24 \pm 0.04$ & $0.46 \pm 0.26$ & $0.12 \pm 0.03$ & $0.03 \pm 0.03$ \\
\hline Pancreas & $24.16 \pm 6.58$ & $0.71 \pm 0.21$ & $0.09 \pm 0.02$ & $0.13 \pm 0.02$ & $0.07 \pm 0.02$ & $0.06 \pm 0.01$ \\
\hline Muscle & $0.97 \pm 0.36$ & $0.11 \pm 0.02$ & $0.09 \pm 0.02$ & $0.06 \pm 0.03$ & $0.03 \pm 0.01$ & $0.03 \pm 0.01$ \\
\hline Bone & $1.84 \pm 0.38$ & $1.29 \pm 0.75$ & $0.58 \pm 0.22$ & $0.48 \pm 0.14$ & $0.18 \pm 0.08$ & $0.15 \pm 0.03$ \\
\hline \multicolumn{7}{|l|}{ Tumour } \\
\hline $\mathrm{rsst}_{2}$ & $22.33 \pm 3.27$ & $29.12 \pm 3.90$ & $3.62 \pm 0.26$ & $22.84 \pm 0.40$ & $13.00 \pm 1.89$ & $7.00 \pm 0.87$ \\
\hline $\mathrm{rsst}_{3}$ & $2.92 \pm 0.91$ & $0.34 \pm 0.06$ & $0.30 \pm 0.04$ & $0.14 \pm 0.03$ & $0.06 \pm 0.03$ & $0.06 \pm 0.02$ \\
\hline
\end{tabular}

\section{Biodistribution studies}

Biodistribution studies of ${ }^{111}$ In-DOTA-sst2-ANT in a dual tumour model bearing HEK293-rsst 2 and HEK293-rsst tumours were carried out from $30 \mathrm{~min}$ up $72 \mathrm{~h}$ p.i. The results are summarized in Tables 1 and 2. At the initial timepoint of the study, ${ }^{111}$ In-DOTA-sst2-ANT had accumulated in the kidneys, the $\mathrm{sst}_{2}$-positive organs including the stomach and pancreas, and into the rsst $_{2}$-expressing tumours. The blood clearance was fast (blood values were reduced by $95 \%$ from $30 \mathrm{~min}$ to $4 \mathrm{~h}$ ) and a fast washout was observed from all nontarget organs, including the stomach and pancreas, where more than $90 \%$ was washed out within $4 \mathrm{~h}$. The accumulation of ${ }^{111}$ In-DOTA-sst2-ANT in the rsst $_{2}$ expressing tumours reached a peak at $4 \mathrm{~h}(29.12 \pm 3.90$ $\% \mathrm{IA} / \mathrm{g})$ and remained high at $24 \mathrm{~h}$ p.i. $(22.84 \pm 0.40 \% \mathrm{IA} /$ $\mathrm{g})$. The washout from the rsst $_{2}$-tumours was relatively slow: about $50 \%$ still remained in the tumour at $48 \mathrm{~h}$ p.i. $(13.00 \pm$ $1.89 \% \mathrm{IA} / \mathrm{g})$ and about $25 \%$ at $72 \mathrm{~h}$ p.i. (7.00 $\pm 0.87 \% \mathrm{IA} / \mathrm{g})$. The specificity of the uptake in rsst $_{2}$-tumours was confirmed by the very low uptake found in rsst $_{3}$-expressing tumours $(0.34 \pm 0.06 \% \mathrm{IA} / \mathrm{g}, 4 \mathrm{~h}$ p.i. $)$ under the same conditions. In addition the blocking experiment performed $4 \mathrm{~h}$ p.i. (coinjection of excess DOTA-sst2-ANT) showed a reduction in

Table 2 Tumour-to-nontumour ratios of ${ }^{111}$ In-DOTA-sst2-ANT in nude mice bearing HEK-rsst ${ }_{2}$ tumours

\begin{tabular}{lccccc}
\hline Ratio & $30 \mathrm{~min}$ & $4 \mathrm{~h}$ & $24 \mathrm{~h}$ & $48 \mathrm{~h}$ & $72 \mathrm{~h}$ \\
\hline Tumour-to-blood & 8.1 & 205 & 440 & 969 & 1199 \\
Tumour-to-muscle & 23 & 263 & 381 & 488 & 233 \\
Tumour-to-liver & 13 & 67 & 72 & 51 & 29 \\
Tumour-to-kidney & 1.0 & 2.8 & 3.1 & & 2.4 \\
\hline
\end{tabular}

tumour uptake by almost $90 \%$, and this was also the case for $s_{2} t_{2}$-expressing tissues, such as the pancreas, while the low uptake in the rsst $_{3}$-expressing tumours remained unaffected. Tumour-to-blood and tumour-to-muscle ratios increased significantly over time, while tumour-to-kidney ratios increasing almost threefold from $30 \mathrm{~min}$ up to $4 \mathrm{~h}$, and remaining unchanged over $48 \mathrm{~h}$.

In order to exclude species differences, biodistribution studies of ${ }^{111}$ In-DOTA-sst2-ANT were performed in mice bearing human sst $_{2}$-expressing tumours (HEK293-hsst ${ }_{2}$ ). The results are summarized in Tables 3 and 4. The biodistribution profile and pharmacokinetics of ${ }^{111}$ In-DOTA-sst2ANT in HEK293-hsst 2 xenografted mice were found to be very similar to those in HEK293-rsst 2 xenografted mice. ${ }^{111}$ In-DOTA-sst2-ANT accumulated in the kidneys, stomach, pancreas (between 7 and $13 \% \mathrm{IA} / \mathrm{g}$ ) and into the hsst $_{2^{-}}$ expressing tumours $(25.26 \pm 4.00 \% \mathrm{IA} / \mathrm{g})$ at $1 \mathrm{~h} \mathrm{p.i.} \mathrm{The}$ uptake in all other organs was much lower (between 0.6 and $2.6 \% \mathrm{IA} / \mathrm{g}$ ). The uptake in the hsst $_{2}$ tumours was the same as in the rsst $_{2}$ tumours, reaching a peak at $4 \mathrm{~h}(29.61 \pm$ $5.13 \% \mathrm{IA} / \mathrm{g}$ ) and showing the same washout rate as described above (see also Fig. 4). The specificity of ${ }^{111} \mathrm{In}$ DOTA-sst2-ANT for the human $\mathrm{sst}_{2}$ receptors was confirmed by blocking experiments, where tumour uptake was reduced by more than $95 \%$ when a 2,000-fold excess of DOTA-sst2-ANT was coinjected.

The biodistribution profile of DOTA-sst2-ANT was additionally evaluated in HEK293-rsst2 xenografted mice using the therapeutic radionuclide ${ }^{177} \mathrm{Lu}$. The biodistribution results of ${ }^{177} \mathrm{Lu}-\mathrm{DOTA}-\mathrm{sst} 2-\mathrm{ANT}$ are summarized in Tables 5 and 6. A higher uptake of ${ }^{177}$ Lu-DOTA-sst2-ANT was observed in some organs, including the pancreas, the adrenals and the gastrointestinal tract (stomach and intestines), where the somatostatin binding sites are located. The 
Table 3 Biodistribution (mean \pm $\mathrm{SD} \% \mathrm{IA} / \mathrm{g}, n=4)$ of ${ }^{111}$ In-DOTAsst2-ANT in nude mice bearing HEK-hsst ${ }_{2}$ tumours
${ }^{\mathrm{a} C o i n j e c t i o n ~ o f ~} 20$ nmol DOTAsst2-ANT.

\begin{tabular}{lrrrrrr}
\hline Organ & \multicolumn{1}{l}{$1 \mathrm{~h}$} & \multicolumn{1}{l}{$4 \mathrm{~h}$} & $4 \mathrm{~h} \mathrm{blocking}^{\mathrm{a}}$ & \multicolumn{1}{l}{$24 \mathrm{~h}$} & \multicolumn{1}{l}{$48 \mathrm{~h}$} & \multicolumn{1}{l}{$72 \mathrm{~h}$} \\
\hline Blood & $1.01 \pm 0.36$ & $0.19 \pm 0.01$ & $0.14 \pm 0.04$ & $0.05 \pm 0.01$ & $0.02 \pm 0.00$ & $0.01 \pm 0.00$ \\
Heart & $0.54 \pm 0.24$ & $0.12 \pm 0.03$ & $0.09 \pm 0.04$ & $0.06 \pm 0.00$ & $0.05 \pm 0.01$ & $0.02 \pm 0.01$ \\
Liver & $1.07 \pm 0.25$ & $0.57 \pm 0.11$ & $0.54 \pm 0.24$ & $0.32 \pm 0.04$ & $0.29 \pm 0.02$ & $0.23 \pm 0.02$ \\
Spleen & $0.65 \pm 0.12$ & $0.25 \pm 0.04$ & $0.24 \pm 0.11$ & $0.16 \pm 0.05$ & $0.13 \pm 0.02$ & $0.12 \pm 0.02$ \\
Lung & $2.60 \pm 0.78$ & $0.50 \pm 0.08$ & $0.38 \pm 0.07$ & $0.15 \pm 0.02$ & $0.12 \pm 0.03$ & $0.10 \pm 0.03$ \\
Kidney & $12.92 \pm 4.12$ & $11.65 \pm 1.44$ & $8.40 \pm 3.58$ & $5.12 \pm 0.78$ & $4.24 \pm 0.27$ & $2.15 \pm 0.31$ \\
Stomach & $7.31 \pm 1.99$ & $0.85 \pm 0.06$ & $0.15 \pm 0.06$ & $0.32 \pm 0.06$ & $0.26 \pm 0.02$ & $0.17 \pm 0.03$ \\
Intestine & $0.80 \pm 0.33$ & $0.18 \pm 0.04$ & $0.12 \pm 0.05$ & $0.08 \pm 0.01$ & $0.06 \pm 0.01$ & $0.04 \pm 0.01$ \\
Adrenals & $1.79 \pm 0.40$ & $0.41 \pm 0.31$ & $0.11 \pm 0.14$ & $0.34 \pm 0.09$ & $0.39 \pm 0.15$ & $0.21 \pm 0.13$ \\
Pancreas & $10.35 \pm 3.33$ & $0.69 \pm 0.08$ & $0.07 \pm 0.01$ & $0.12 \pm 0.01$ & $0.08 \pm 0.02$ & $0.05 \pm 0.02$ \\
Muscle & $0.38 \pm 0.06$ & $0.11 \pm 0.02$ & $0.05 \pm 0.03$ & $0.06 \pm 0.02$ & $0.05 \pm 0.02$ & $0.03 \pm 0.02$ \\
Bone & $1.92 \pm 1.60$ & $0.61 \pm 0.39$ & $0.02 \pm 0.07$ & $0.39 \pm 0.19$ & $0.46 \pm 0.45$ & $0.19 \pm 0.18$ \\
Tumour & $25.26 \pm 4.00$ & $29.61 \pm 5.13$ & $1.70 \pm 0.17$ & $19.67 \pm 3.39$ & $12.41 \pm 0.69$ & $7.02 \pm 0.28$ \\
\hline
\end{tabular}

uptake of ${ }^{177}$ Lu-DOTA-sst2-ANT in rsst $_{2}$-tumours was slightly higher at $4 \mathrm{~h}$ p.i. $(34.90 \pm 2.86 \% \mathrm{IA} / \mathrm{g})$ than that of ${ }^{111}$ In-DOTA-sst2-ANT, but the difference was not statistically significant $(p>0.05)$. The specificity of ${ }^{177}$ Lu-DOTAsst2-ANT for the $\mathrm{rsst}_{2}$ was also confirmed by blocking experiments with coinjection of a 2,000-fold excess of DOTA-sst2-ANT which led to a reduction of more than $95 \%$ of the radioactivity in the tumour. The washout of ${ }^{177} \mathrm{Lu}$-DOTA-sst2-ANT from the tumour was relatively slow and very similar to that of ${ }^{111}$ In-DOTA-sst2-ANT.

\section{Blocking of renal uptake}

The influence of Gelofusine and lysine on renal uptake of ${ }^{111}$ In-DOTA-sst2-ANT in HEK293-rsst 2 xenografted mice was studied in comparison to mice without any intervention $24 \mathrm{~h}$ p.i. The biodistribution results are summarized in Fig. 5. Injection of lysine, Gelofusine or PBS (control group) resulted in similar ${ }^{111}$ In-DOTA-sst2-ANT concentrations in all tissues and organs except for kidneys. Compared to the control group, intravenous administration of $4 \mathrm{mg}$ of Gelofusine reduced kidney uptake from $7.38 \pm 0.09 \% \mathrm{IA} / \mathrm{g}$ (control group) to $4.36 \pm 0.27 \% \mathrm{IA} / \mathrm{g}$. Similarly, the administration of $20 \mathrm{mg}$ lysine reduced kidney uptake from $7.38 \pm$ $0.09 \% \mathrm{IA} / \mathrm{g}$ (control group) to $3.84 \pm 0.27 \% \mathrm{IA} / \mathrm{g}$. The use of higher injected doses of Gelofusine $(6 \mathrm{mg})$ and lysine

Table 4 Tumour-to-nontumour ratios of ${ }^{111}$ In-DOTA-sst2-ANT in nude mice bearing HEK-hsst ${ }_{2}$ tumours

\begin{tabular}{lcrrrr}
\hline Ratio & $1 \mathrm{~h}$ & $4 \mathrm{~h}$ & $24 \mathrm{~h}$ & $48 \mathrm{~h}$ & $72 \mathrm{~h}$ \\
\hline Tumour-to-blood & 25 & 153 & 411 & 596 & 742 \\
Tumour-to-muscle & 67 & 279 & 348 & 275 & 266 \\
Tumour-to-liver & 24 & 52 & 61 & 43 & 31 \\
Tumour-to-kidney & 1.9 & 2.5 & 3.8 & 2.9 & 3.3 \\
\hline
\end{tabular}

(30 $\mathrm{mg})$ resulted in a similar reduction in kidney uptake (3.58 \pm 0.44 and $3.69 \pm 0.44 \% \mathrm{IA} / \mathrm{g}$, respectively). No statistically significant difference was found between animals injected with lysine and those injected with Gelofusine. The injection of lysine or Gelofusine did not affect the tumour uptake resulting in an increase of tumour-to-kidney ratio by a factor of 2 .

\section{Discussion}

We have recently shown that the somatostatin-based radioantagonist ${ }^{111}$ In-DOTA-sst2-ANT shows superior pharmacokinetic properties to the agonists ${ }^{111}$ In-DTPA-TATE and ${ }^{111}$ In-DOTA-NOC in rat sst2 xenografted mice [6]. We found not only higher tumour uptake but also surprisingly high retention over $24 \mathrm{~h}$. The same pharmacokinetics were not found with ${ }^{64} \mathrm{Cu}-\mathrm{CB}-\mathrm{TE} 2 \mathrm{~A}-\mathrm{sst} 2-\mathrm{ANT}$ compared with

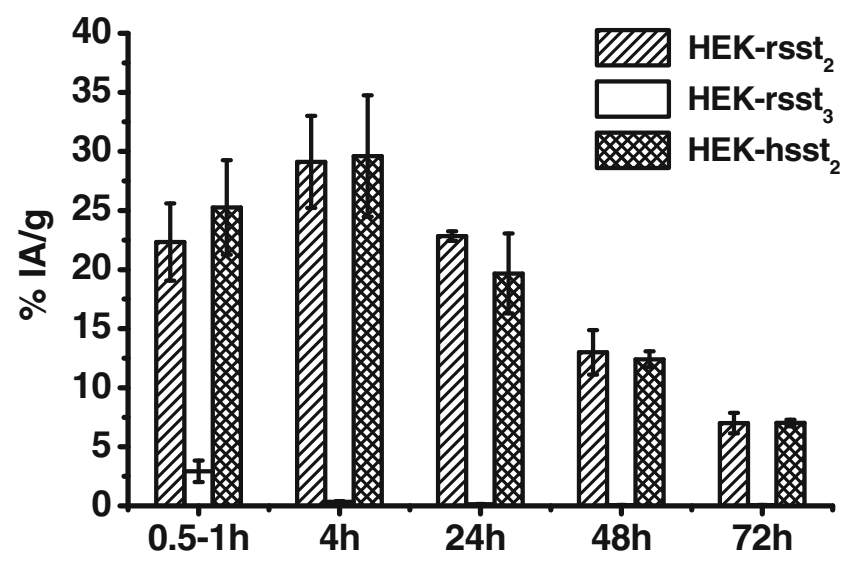

Fig. 4 In vivo accumulation and washout of ${ }^{111}$ In-DOTA-sst2-ANT over time in HEK293 cells expressing different sst receptors ( $r s s t_{2}$ rat sst $_{2}$, rsst $_{3}$ rat sst $_{3}$, hsst $_{2}$ human sst $_{2}$ ). Results are presented as mean \pm SD \%IA/g 
Table 5 Biodistribution (mean \pm SD $\%$ IA $/ \mathrm{g}, n=4)$ of ${ }^{177}$

Lu-DOTA-sst2-ANT in nude mice bearing $\mathrm{HEK}-\mathrm{rsst}_{2}$ tumours

${ }^{\mathrm{a}}$ Coinjection of $20 \mathrm{nmol}$ DOTAsst2-ANT.

\begin{tabular}{lrrrrr}
\hline Organ & \multicolumn{1}{l}{$4 \mathrm{~h}$} & $4 \mathrm{~h} \mathrm{blocking}^{\mathrm{a}}$ & \multicolumn{1}{l}{$24 \mathrm{~h}$} & \multicolumn{1}{l}{$48 \mathrm{~h}$} & \multicolumn{1}{l}{$72 \mathrm{~h}$} \\
\hline Blood & $0.20 \pm 0.02$ & $0.31 \pm 0.05$ & $0.06 \pm 0.01$ & $0.03 \pm 0.00$ & $0.02 \pm 0.00$ \\
Heart & $0.25 \pm 0.03$ & $0.12 \pm 0.04$ & $0.08 \pm 0.03$ & $0.05 \pm 0.01$ & $0.02 \pm 0.01$ \\
Liver & $0.80 \pm 0.07$ & $0.86 \pm 0.10$ & $0.40 \pm 0.09$ & $0.30 \pm 0.02$ & $0.24 \pm 0.05$ \\
Spleen & $1.03 \pm 0.86$ & $0.34 \pm 0.05$ & $0.23 \pm 0.05$ & $0.14 \pm 0.01$ & $0.12 \pm 0.03$ \\
Lung & $2.88 \pm 1.54$ & $0.54 \pm 0.10$ & $0.44 \pm 0.13$ & $0.19 \pm 0.03$ & $0.08 \pm 0.03$ \\
Kidney & $10.82 \pm 1.76$ & $13.91 \pm 1.06$ & $6.03 \pm 1.49$ & $3.35 \pm 0.56$ & $1.70 \pm 0.35$ \\
Stomach & $17.26 \pm 2.39$ & $0.32 \pm 0.08$ & $1.83 \pm 0.59$ & $0.77 \pm 0.02$ & $0.39 \pm 0.02$ \\
Intestine & $0.96 \pm 0.37$ & $0.18 \pm 0.05$ & $0.28 \pm 0.09$ & $0.07 \pm 0.02$ & $0.05 \pm 0.01$ \\
Adrenals & $2.85 \pm 1.04$ & $0.40 \pm 0.25$ & $1.37 \pm 0.71$ & $0.48 \pm 0.03$ & $0.04 \pm 0.03$ \\
Pancreas & $26.11 \pm 7.63$ & $0.21 \pm 0.01$ & $1.18 \pm 0.46$ & $0.37 \pm 0.02$ & $0.26 \pm 0.01$ \\
Muscle & $0.22 \pm 0.07$ & $0.10 \pm 0.04$ & $0.15 \pm 0.07$ & $0.04 \pm 0.01$ & $0.03 \pm 0.02$ \\
Bone & $1.06 \pm 0.13$ & $0.05 \pm 0.04$ & $0.92 \pm 0.27$ & $0.36 \pm 0.17$ & $0.44 \pm 0.08$ \\
Tumour & $34.90 \pm 2.86$ & $1.33 \pm 0.19$ & $25.65 \pm 6.37$ & $13.57 \pm 2.17$ & $7.79 \pm 0.68$ \\
\hline
\end{tabular}

the agonist ${ }^{64} \mathrm{Cu}-\mathrm{CB}-\mathrm{TE} 2 \mathrm{~A}-\mathrm{TATE}$ in nude mice xenografted with the rat pancreatic tumour cell line AR42J, despite the fact that the authors found a 14-fold higher number of receptor binding sites for the antagonist [7]. As somatostatin-based radiopeptides are the only family of radiopeptides having an impact on patient care as diagnostic agents (SPECT and PET) [11] and in targeted radionuclide therapy of neuroendocrine tumours [12, 13], we reasoned that any improvement in this field is worthwhile, and in particular radioantagonists deserve more scrutiny. We therefore studied this peptide more thoroughly. In the present study we evaluated extensively the performance of ${ }^{111}$ InDOTA-sst2-ANT in cell cultures in vitro and extended the evaluation of its biodistribution to later time-points. This was also done in a xenograft model expressing the human sst2 receptor to ensure that there were no species differences as found with radiopeptides targeting the gastrin-releasing peptide receptor [8]. In addition, we studied the pharmacokinetics of ${ }^{177}$ Lu-DOTA-sst2-ANT to exclude any potential influence of the radiometal on pharmacological properties and to determine the potential usefulness of this radioantagonist in therapeutic applications. We also studied the blocking of kidney uptake with blocking agents used clinically.

There are several important results from these studies. The pharmacokinetic data of ${ }^{111}$ In-DOTA-sst2-ANT clearly

Table 6 Tumour-to-nontumour ratios of ${ }^{177}$ Lu-DOTA-sst2-ANT in nude mice bearing HEK-rsst ${ }_{2}$ tumours

\begin{tabular}{lcccc}
\hline Ratio & $4 \mathrm{~h}$ & $24 \mathrm{~h}$ & $48 \mathrm{~h}$ & $72 \mathrm{~h}$ \\
\hline Tumour-to-blood & 175 & 428 & 452 & 390 \\
Tumour-to-muscle & 159 & 171 & 339 & 260 \\
Tumour-to-liver & 44 & 64 & 45 & 33 \\
Tumour-to-kidney & 3.2 & 4.3 & 4.1 & 4.6 \\
\hline
\end{tabular}

showed that there was no significant difference between human and rat receptor with regard to tumour uptake and washout from the tumour resulting in an almost identical area under the curve. This may be explained by the similar $B_{\text {max }}$ values (data not shown) and the high homology of the human and the rat $\mathrm{sst}_{2}$ receptor $[14,15] .{ }^{177}$ Lu-DOTA-sst2ANT was studied in comparison with ${ }^{111}$ In-DOTA-sst2ANT up to $72 \mathrm{~h}$. In agreement with the results from the saturation binding studies demonstrating a similar dissociation constant $\left(K_{\mathrm{d}}\right)$ for both (radio)metal-labelled peptides, the tumour uptake was almost identical for the two radiopeptides. This indicates no influence of the radiometal on this DOTA-conjugated antagonist rendering ${ }^{177}$ Lu-DOTAsst2-ANT a promising radiotherapeutic agent. This result is

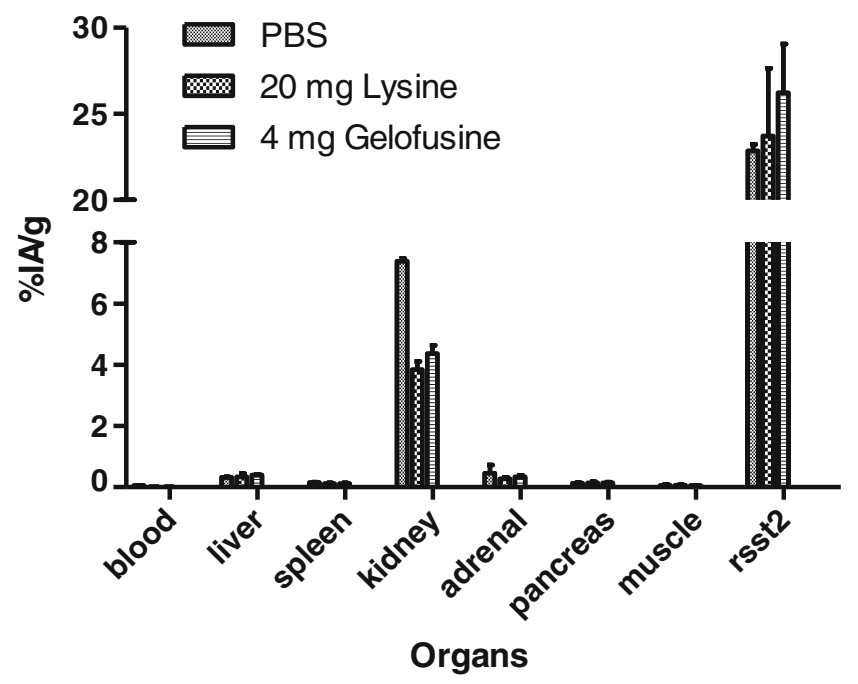

Fig. 5 Biodistribution of ${ }^{111}$ In-DOTA-sst2-ANT in nude mice bearing HEK293-rsst ${ }_{2}$ tumours. Three groups of mice $(n=3)$ received lysine (20 mg) or Gelofusine (4 mg) or PBS (control group) 5 min before the injection of the radiopeptide. Biodistribution was evaluated $24 \mathrm{~h}$ p.i. and the results are expressed as mean $\pm \mathrm{SD} \% \mathrm{IA} / \mathrm{g}(n=3)$ 
somewhat contrary to the result with another somatostatinbased radioantagonist [16] when we found distinct differences in pharmacology depending on radiometal and chelator. The specificity of the radiopeptides in the HEK293-rsst2 and HEK293-hsst2 tumours as well as in sst $_{2}$-positive tissues, such as the pancreas, was clearly demonstrated by blocking studies where $>90 \%$ blocking was achieved in all experiments. In an additional study to confirm specificity a dual tumour model, HEK293-rsst ${ }_{3}$ in one flank and HEK293-rsst ${ }_{2}$ in the other, was used. The uptake in HEK293-rsst ${ }_{3}$ tumours was negligible at time-points more than $4 \mathrm{~h}$ p.i. Blood clearance of both radiopeptides was fast resulting in high tumour-to-blood and tumour-to-normal organ ratios at early time-points which increased with time for most organs. The blocking agent did not affect the uptake in the kidneys, indicating that this uptake is not receptor-mediated. Gelofusine and lysine can efficiently reduce the renal uptake of radiopeptides [17, 18] probably via a different mechanism. Kidney blocking with lysine and Gelofusine was effective, as expected. Neither agent blocked tumour uptake but did block kidney uptake by about $50 \%$, increasing the tumour-to-kidney ratio about twofold.

An open question concerning internalization was the discrepancy - although low - between the lack of internalization found when receptor trafficking is studied using immunofluorescence microscopy [6] compared to radioligand internalization. In immunofluorescence microscopy-

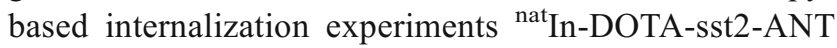
did not trigger $s_{2} t_{2}$ receptor internalization [6], while in the radioligand internalization experiments a low internalization rate was found for the ${ }^{111}$ In-DOTA-sst2-ANT. It should be considered that in the immunofluorescence microscopy experiments, where receptor trafficking is monitored, the ligand is used in high excess while in the radioligand internalization experiments the radiopeptide concentration is far below tumour saturation. Concerning the internalization of radiolabelled antagonists similar observations have been made by others. A low degree of internalization was also detected when ${ }^{64} \mathrm{Cu}-\mathrm{CB}$ TE2A-sst2-ANT was studied in AR42J cells [7] and in sst $_{2}$-positive HCT116 cells [19]. In the present study, no radiopeptide internalization in HEK293 cells not expressing $\mathrm{sst}_{2}$ was found. Additionally, pretreatment of the HEK293-rsst ${ }_{2}$ cells with hypertonic sucrose, which is known to prevent receptor endocytosis by preventing clathrin-coated pit formation [20], distinctly reduced radiopeptide internalization. These findings are strong indications of a specific receptor-mediated internalization process. Why this is not observable if receptor trafficking is studied is not clear at the moment, but may be a consequence of the different experimental conditions described above.
The in vitro dissociation experiment was performed to determine if the slow dissociation of the radiopeptide is the explanation for the long in vivo tumour residence time. This was found to be not the case as the residence half-life in the cell culture experiment was about $10 \mathrm{~min}$. Approximately $90 \%$ of the radiopeptide dissociated within $60 \mathrm{~min}$, while the remaining $10 \%$ was internalized. Therefore, we conclude that microenvironmental phenomena including the chaotic tumour vessel system are responsible for the long retention.

Although obtained in a very small number of patients, the preclinical data showing the superiority of radiolabelled somatostatin-based antagonists over agonists seem to be translatable to the clinic. ${ }^{111}$ In-DOTAsst2-ANT was studied in an early human use study in comparison with ${ }^{111}$ In-DTPA-octreotide (OctreoScan ${ }^{\circledR}$ ) in five patients with (neuro)endocrine tumours [21]. Tumour uptake and in particular the tumour-to-kidney ratio was clearly in favour of ${ }^{111}$ In-DOTA-sst2-ANT. This result is promising and gives hope for improved diagnostic (SPECT and PET) and therapeutic vectors targeting sst-positive tumours.

In summary, the radiopeptide ${ }^{111}$ In-DOTA-sst2-ANT was previously studied and showed superior pharmacokinetics if compared to potent agonists. The study reported here extends the previous studies to new tumour models, the ${ }^{177} \mathrm{Lu}$-labelled version and extension of the pharmacokinetics to later time-points. The data show that ${ }^{111} \mathrm{In}-/{ }^{177} \mathrm{Lu}$ DOTA-sst2-ANT are excellent sst $_{2}$-targeting agents which deserve translation to trials in patients and indicate the relevance of these radiopeptides not only in diagnostic imaging but also in targeted radionuclide therapy of sstpositive tumours.

Acknowledgments We thank the Swiss National Science Foundation and the FP7 project TARCC for financial support, and also the COST action D38.

Conflicts of interest None.

\section{References}

1. Reubi JC, Maecke HR. Peptide-based probes for cancer imaging. J Nucl Med. 2008;49:1735-8.

2. Reubi JC. Peptide receptors as molecular targets for cancer diagnosis and therapy. Endocr Rev. 2003;24:389-427.

3. Storch D, Behe M, Walter MA, Chen J, Powell P, Mikolajczak R, et al. Evaluation of $[99 \mathrm{mTc} / \mathrm{EDDA} / \mathrm{HYNIC0}]$ octreotide derivatives compared with [111In-DOTA0, Tyr3, Thr8]octreotide and [111In-DTPA0]octreotide: does tumor or pancreas uptake correlate with the rate of internalization? J Nucl Med. 2005;46:1561-9.

4. Fani M, Mueller A, Tamma ML, Nicolas G, Rink HR, Cescato R, et al. Radiolabeled bicyclic somatostatin-based analogs: a novel class of potential radiotracers for SPECT/PET of neuroendocrine tumors. J Nucl Med. 2010;51:1771-9. 
5. Ginj M, Zhang H, Eisenwiener KP, Wild D, Schulz S, Rink H, et al. New pansomatostatin ligands and their chelated versions: affinity profile, agonist activity, internalization, and tumor targeting. Clin Cancer Res. 2008;14:2019-27.

6. Ginj M, Zhang H, Waser B, Cescato R, Wild D, Wang X, et al. Radiolabeled somatostatin receptor antagonists are preferable to agonists for in vivo peptide receptor targeting of tumors. Proc Natl Acad Sci U S A. 2006;103:16436-41.

7. Wadas TJ, Eiblmaier M, Zheleznyak A, Sherman CD, Ferdani R, Liang K, et al. Preparation and biological evaluation of $64 \mathrm{Cu}-\mathrm{CB}-$ TE2A-sst2-ANT, a somatostatin antagonist for PET imaging of somatostatin receptor-positive tumors. J Nucl Med. 2008;49:1819-27.

8. Maina T, Nock BA, Zhang H, Nikolopoulou A, Waser B, Reubi JC, et al. Species differences of bombesin analog interactions with GRP-R define the choice of animal models in the development of GRP-R-targeting drugs. J Nucl Med. 2005;46:823-30.

9. Cescato R, Erchegyi J, Waser B, Piccand V, Maecke HR, Rivier $\mathrm{JE}$, et al. Design and in vitro characterization of highly sst2selective somatostatin antagonists suitable for radiotargeting. J Med Chem. 2008;51:4030-7.

10. Ginj M, Schmitt JS, Chen J, Waser B, Reubi JC, de Jong M, et al. Design, synthesis, and biological evaluation of somatostatin-based radiopeptides. Chem Biol. 2006;13:1081-90.

11. Maecke HR, Reubi JC. Somatostatin receptors as targets for nuclear medicine imaging and radionuclide treatment. J Nucl Med. 2011;52:841-4.

12. Imhof A, Brunner P, Marincek N, Briel M, Schindler C, Rasch H, et al. Response, survival, and long-term toxicity after therapy with the radiolabeled somatostatin analogue [90Y-DOTA]-TOC in metastasized neuroendocrine cancers. J Clin Oncol. 2011;29:2416-23.
13. Kwekkeboom DJ, de Herder WW, Kam BL, van Eijck CH, van Essen M, Kooij PP, et al. Treatment with the radiolabeled somatostatin analog [177Lu-DOTA0,Tyr3]octreotate: toxicity, efficacy, and survival. J Clin Oncol. 2008;26:2124-30.

14. Weckbecker G, Lewis I, Albert R, Schmid HA, Hoyer D, Bruns C. Opportunities in somatostatin research: biological, chemical and therapeutic aspects. Nat Rev Drug Discov. 2003;2:999-1017.

15. Meyerhof W. The elucidation of somatostatin receptor functions: a current view. Rev Physiol Biochem Pharmacol. 1998;133:55-108.

16. Fani M, Del Pozzo L, Abiraj K, Mansi R, Tamma ML, Cescato R, et al. PET of somatostatin receptor-positive tumors using $64 \mathrm{Cu}-$ and $68 \mathrm{Ga}$-somatostatin antagonists: the chelate makes the difference. J Nucl Med. 2011;52:1110-8.

17. van Eerd JE, Vegt E, Wetzels JF, Russel FG, Masereeuw R, Corstens FH, et al. Gelatin-based plasma expander effectively reduces renal uptake of $111 \mathrm{In}$-octreotide in mice and rats. J Nucl Med. 2006;47:528-33.

18. Vegt E, Wetzels JF, Russel FG, Masereeuw R, Boerman OC, van Eerd JE, et al. Renal uptake of radiolabeled octreotide in human subjects is efficiently inhibited by succinylated gelatin. J Nucl Med. 2006;47:432-6.

19. Nguyen K, Parry JJ, Rogers BE, Anderson CJ. Evaluation of copper-64-labeled somatostatin agonists and antagonist in SSTr2transfected cell lines that are positive and negative for p53: implications for cancer therapy. Nucl Med Biol. 2012;39:187-97.

20. Heuser JE, Anderson RG. Hypertonic media inhibit receptormediated endocytosis by blocking clathrin-coated pit formation. J Cell Biol. 1989;108:389-400.

21. Wild D, Fani M, Behe M, Brink I, Rivier JE, Reubi JC, et al. First clinical evidence that imaging with somatostatin receptor antagonists is feasible. J Nucl Med. 2011;52:1412-7. 